\title{
A Case of Multiple Cutaneous Metastases from Hepatocellular Carcinoma Mimicking Pyogenic Granuloma
}

Obara $\mathrm{K}^{*}$ and Amoh $\mathrm{Y}$

Department of Dermatology, Kitasato University School of Medicine, Kanagawa, Japan

\begin{abstract}
Hepatocellular carcinoma (HCC) metastasizing to the skin is uncommon and carries a poor prognosis. The most frequent sites of skin metastasis are the face and scalp. Cutaneous metastatic lesions usually appear as nodules, but sometimes show a pyogenic granuloma-like morphology. We report a case of multiple skin metastases from HCC that resembled pyogenic granulomas, and showing HepPar1 as a specific marker of HCC. A key feature in this case was metastasis to the mandibular gingiva and digits. This case emphasizes the need to recognize that gingival and digital masses like benign or inflammatory lesions might represent an initial sign of HCC.
\end{abstract}

Keywords: Hepatocellular carcinoma; Pyogenic granuloma; Gingival; Acrometastasis; Hepatocyte paraffin-1

\section{Introduction}

The most frequent sites of skin metastasis from hepatocellular carcinoma (HCC) are the face and scalp, with lesions appearing as single or multiple, firm, painless, nonulcerative, reddish nodules, measuring 1-2.5 cm in diameter. These metastases represent a sign of poor prognosis, indicating the strong possibility of metastases in other regions of the body, and point to a median survival time of less than 5 months [1]. We report a case of multiple skin metastases from HCC that resembled pyogenic granuloma, including the mandibular gingiva and digits.

\section{Case Report}

An 86-year-old Japanese man presented with easy bleeding from a cutaneous nodule on the left elbow that had been present for 1 month (Figure 1). The nodule resembled pyogenic granuloma, measured $10 \mathrm{~mm}$ in diameter, and was solitary, soft, berry-shaped and reddish. Medical history included a 6-year history of HCC, originating from liver cirrhosis associated with chronic hepatitis C. He had undergone partial hepatectomy and radiofrequency ablation as initial treatment. The patient remained free of clinical disease for slightly more than 4 years, from which point recurrent HCC in the liver was diagnosed. Treatment for recurrences included transcatheter arterial embolization twice and radiofrequency ablation 3 times. Laboratory investigations revealed slightly elevated serum levels of protein induced by vitamin $\mathrm{K}$ absence or antagonist II (PIVKA-II) $(93 \mathrm{mAU} / \mathrm{ml}$; normal range, $0-40 \mathrm{mAU} / \mathrm{ml}$ ). No local recurrence or distant metastasis was evident on computed tomography. We initially suspected pyogenic granuloma based on the clinical appearance. The nodule was removed and sent for histopathological examination.

Histologically, eosinophilic neoplastic cells proliferated with a trabecular pattern in the dermis (Figures 2a-2d). The mass consisted of irregular columns of large, polygonal cells and intervening capillaries forming sinusoid-like structures. Tumor cells resembling hepatocytes with nuclear atypia showed positive results for hepatocyte paraffin-1 (HepPar1) and CD10 on immunohistochemical examination. Negative results were obtained for alpha-fetoprotein antibodies and carcinoembryonic antigen.

From these clinical and histopathological findings, we diagnosed cutaneous metastasis from HCC. After 6 months of follow-up, the patient developed local recurrence of HCC and multiple metastases to the face, mandibular gingiva and digits (Figures 3a-3c). PIVKA-II

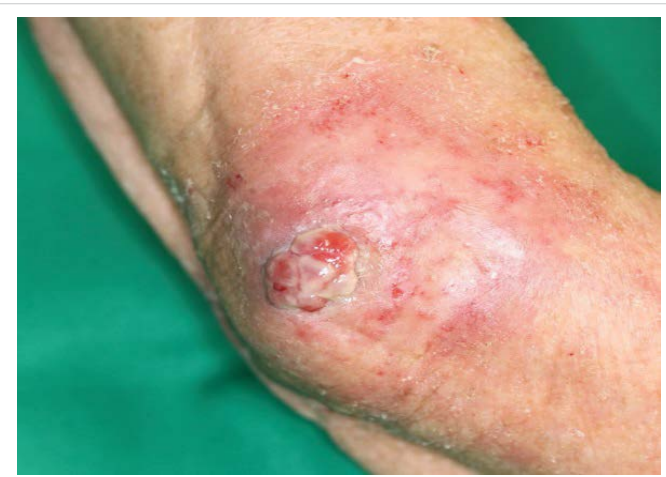

Figure 1:Soft, reddish nodule resembling pyogenic granuloma on the left elbow.

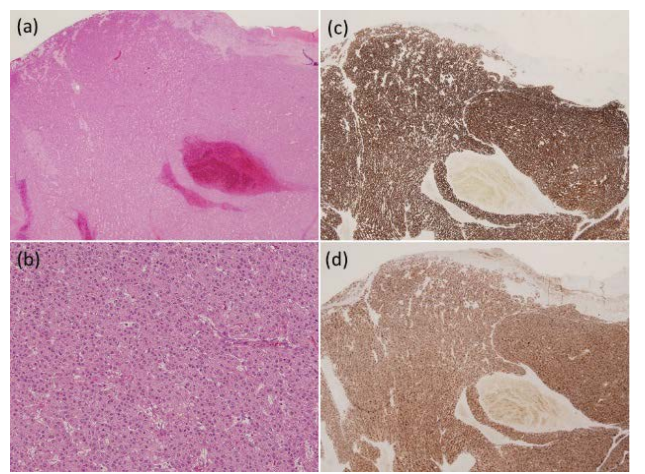

Figure 2: (a) Histological features of the nodule on the left elbow: Eosinophilic neoplastic cells proliferate in a trabecular pattern within the dermis (hematoxylin and eosin stain ( $H$ and $E$ ), original magnification, 40x). (b) The mass consists of irregular columns of large, polygonal cells with nuclear atypia and intervening capillaries forming sinusoid-like structures ( $H$ and $E, 200 \times)$. (c, d) Immunohistochemical analysis indicates that tumor cells express HepPar1 (c) and CD10 (d) (original magnification, 40x).

*Corresponding author: Obara K, Department of Dermatology, Kitasato University School of Medicine, Kanagawa, Japan, Tel: +81-427-78-8468; E-mail: obarakoya@yahoo.co.jp

Received June 01, 2018; Accepted June 05, 2018; Published June 08, 2018

Citation: Obara K, Amoh Y (2018) A Case of Multiple Cutaneous Metastases from Hepatocellular Carcinoma Mimicking Pyogenic Granuloma. J Clin Case Rep 8: 1126. doi: $10.4172 / 2165-7920.10001126$

Copyright: @ 2018 Obara K, et al. This is an open-access article distributed under the terms of the Creative Commons Attribution License, which permits unrestricted use, distribution, and reproduction in any medium, provided the original author and source are credited. 


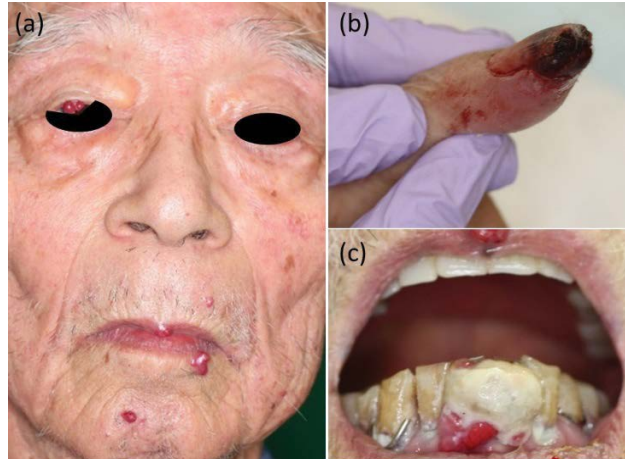

Figure 3: Multiple cutaneous metastases from HCC on the face (a) mandibular gingiva (b) and digits (c).

concentration increased significantly to $20,651 \mathrm{mAU} / \mathrm{ml}$. The patient underwent partial removal of the metastatic lesions for functional (e.g., feeding) and hygiene improvements. A month later, the patient died from cutaneous and bone metastatic spread of the tumor.

\section{Discussion}

Metastasis from HCC occurs in $55 \%$ to $70 \%$ of cases, most frequently to the lungs, lymph nodes and adrenal glands, with skin metastases accounting for only $0.2 \%$ to $2.7 \%$ [2]. Cutaneous metastatic lesions usually appear as nodules, but sometimes show a pyogenic granulomalike or hemangiomatous morphology with easy bleeding. In addition to HCC, renal cell carcinoma and prostate carcinoma also metastasize to the skin with similar manifestations $[3,4]$. The pathological diagnosis of metastatic $\mathrm{HCC}$ at an extrahepatic metastatic site can be made relatively easily in patients with HCC. The histology of HCC shows trabecular and pseudo glandular patterns comprising acidophilic cells. Detection of bile is confirmative of HCC. Mallory bodies strongly suggest HCC. However, immunohistochemical studies are of great value, and HepPar 1 is a relatively specific marker of hepatocytes and HCC [5].

The key feature of our case was metastasis to the mandibular gingiva and digits from HCC. Metastatic carcinoma to the jaws and oral region is rare and accounts for approximately $1 \%$ of all malignant oral tumors [6]. The most common sites were the breast $(21.8 \%)$, followed by the lung (12.6\%), adrenal glands (8.7\%) and kidneys (7.9\%) [7]. McDaniel et al., observed only 1 (3\%) of the 32 cases of HCC metastasis to the oral region [8]. In cases of metastasis to the oral cavity, mean survival time was 7 months [9]. The mandible is the most common location for metastasis, with the molar area being the most frequently involved site. Blood vessels in the posterior region of the mandible are well developed, which might help tumor cells to become entrenched [6].

Acrometastasis, representing metastasis to the hands or feet, comprises only $0.1 \%$ of all metastases [10]. The most common primary site was the lung (44\%), followed by kidney (12\%), breast (10\%) and colon cancers (6\%) [11]. Afshar et al., observed only 8 (4\%) of 221 cases of HCC metastasis to the hand and wrist [12]. The median survival of patients in the reported cases was 6 months [11]. The mechanisms underlying acrometastasis remain poorly understood. The phalangeal absence of bone marrow has led to alternative theories regarding hematologic spread, including increased blood flow and trauma [13]. One suggestion is that the prostaglandins released following a traumatic experience may be responsible for cell migration and adhesion to bone [14].

\section{Conclusion}

Skin metastasis from internal neoplasms should be considered among the differential diagnoses in the evaluation of cutaneous tumors. In particular, this case emphasizes the need to recognize that gingival and digital masses resembling benign or inflammatory lesions such as pyogenic granuloma might represent an initial sign of HCC.

\section{References}

1. Okusaka T, Okada S, Ishii H, Nose H, Nagahama H, et al. (1997) Prognosis of hepatocellular carcinoma patients with extrahepatic metastases. Hepatogastroenterol 44: 251-257.

2. Okuda K (1992) Hepatocellular carcinoma: Recent progress. Hepatol 15: 948-963.

3. Jin WW, Chung JM, Jung KE, Park JW, Kim MH (2008) A case of metastatic renal cell carcinoma mimicking granuloma pyogenicum. Ann Dermatol 20: 263-266.

4. Powell FC, Venencie PY, Winkelmann RK (1984) Metastatic prostate carcinoma manifesting as penile nodules. Arch Dermatol 120: 1604-1606.

5. Lugli A, Tornillo L, Miriacher M, Bundi M, Sauter G, et al. (2004) Hepatocyte paraffin 1 expression in human normal and neoplastic tissues: Tissue microarray analysis of 3,940 tissue samples. Am J Clin Pathol 122: 721-727.

6. Lee YH, Lee $\mathrm{Jl}$ (2017) Metastatic carcinoma of the oral region: An analysis of 21 cases. Med Oral Patol Oral Cir Bucal 22: 359-365.

7. Hirshberg A, Leibovich P, Buchner A (1994) Metastatic tumors to the jaw bones: Analysis of 390 cases. J Oral Pathol Med 23: 337-341.

8. McDaniel RK, Luna MA, Stimson PG (1971) Metastatic tumors in the jaws. Oral Surg Oral Med Oral Pathol 31: 380-386

9. Sánchez-Jiménez J, Acebal-Blanco F, Arévalo-Arévalo RE, Molina-Martínez M (2005) Metastatic tumours in upper maxillary bone of esophagea adenocarcinoma. A case reports. Med Oral Patol Oral Cir Bucal 10: 252-257.

10. Kerin R (1987) The hand in metastatic disease. J Hand Surg Am 12: 77-83.

11. Flynn CJ, Danjoux C, Wong J, Christakis M, Rubenstein J, et al. (2008) Two cases of acrometastasis to the hands and review of the literature. Curr Oncol 15: $51-58$

12. Afshar A, Farhadnia $P$, Khalkhali $H$ (2014) Metastases to the hand and wrist: An analysis of 221 cases. J Hand Surg Am 39: 923-932.

13. Healey JH, Turnbull AD, Miedema B, Lane JM (1986) Acrometastases: A study of twenty-nine patients with osseous involvement of the hands and feet. J Bone Joint Surg Am 68: 743-746.

14. Tolo ET, Cooney WP, Wenger DE (2002) Renal cell carcinoma with metastases to the triquetrum: Case report. J Hand Surg Am 27: 876-881. 